\title{
Prescripción en Cascada y Desprescripción
}

\author{
Francisco Tomás Pagán Núñez ${ }^{a}$, Francisco Tejada Cifuentes ${ }^{b}$
}

\begin{abstract}
a Farmacéutico Especialista en Farmacia Hospitalaria.

${ }^{\mathrm{b}}$ Doctor en Farmacia.

Especialista en Análisis Clínicos. Farmacéutico de Atención Primaria.

Correspondencia: Francisco Tejada Cifuentes. Gerencia de Atención Primaria. C/ Dionisio Guardiola 17, 02001, Albacete. Telf.: 967510825.

Correo electrónico:

ftejada@sescam.jccm.es

Recibido el 27 de enero de 2012.
\end{abstract}

Aceptado para su publicación el 28 de marzo de 2012

\begin{abstract}
RESUMEN
La prescripción en cascada se produce cuando un nuevo fármaco es prescrito para "tratar" una reacción adversa producida por otro fármaco, basándose en la creencia errónea de que una nueva condición médica se ha desarrollado. Los acontecimientos adversos asociados a la prescripción en cascada se producen cuando el segundo fármaco aumenta la severidad de la reacción adversa producida por el primer fármaco, o cuando el segundo fármaco expone al paciente a la aparición de nuevas reacciones adversas. La clave para prevenir la prescripción en cascada reside en la prevención y rápida detección de las reacciones adversas. Los profesionales de la salud deben ser capaces de reconocer cuándo un medicamento debe ser retirado y cómo hacerlo.

La desprescripción debe considerarse cuando existe polimedicación, reacciones adversas a medicamentos, ineficacia del tratamiento, caídas o cuando los objetivos del tratamiento han cambiado. Un enfoque cauteloso de la desprescripción incluye dos principios: retirar los fármacos de uno en uno y disminuir las dosis gradualmente durante semanas o meses.

Palabras clave. Cascada de prescripción, Reacción Adversa, Polifarmacia, Retirada de fármacos.
\end{abstract}

\section{ABSTRACT}

A prescribing cascade occurs when a new medicine is prescribed to 'treat' an adverse reaction to another drug in the mistaken belief that a new medical condition requiring treatment has developed. Adverse outcomes associated with prescribing cascades can result when the second drug increases the severity of the adverse reaction to the first drug or when the second drug places the patient at risk of additional adverse drug reactions. The key to preventing prescribing cascades lies in the avoidance and early detection of adverse drug reactions and an increased awareness and recognition of the potential for adverse reactions.

Medicines have adverse effects and the use of multiple medicines, polypharmacy, can be associated with poorer outcomes. Health professionals need to recognise when medicines should be ceased and how to deprescribe.

Deprescribing could be considered when there is polypharmacy, adverse drug reactions, ineffective treatment, falls or when treatment goals have changed. If patients are slowly weaned off their medicines, withdrawal and rebound syndromes are usually not serious. A cautious approach to deprescribing includes two principles - stop one drug at a time and wean doses slowly over weeks and months.

Key words. Prescribing cascade, adverse effects, polypharmacy, drug withdrawal.

\section{CASCADA DE PRESCRIPCIÓN}

Los eventos adversos asociados con el uso de los medicamentos son comunes y representan una carga significativa sobre el sistema de salud, tanto en términos de resultados de salud como de aumento de costes. Se estima que el $10 \%$ de pacientes que acuden a consultas de medicina general han tenido un efecto adverso relacionado con medicamentos en los últimos seis meses $^{1}$. Los eventos adversos relacionados con medicamentos incluyen los errores en la forma en que el medicamento se utiliza y las reacciones adversas que resultan de las propiedades farmacológicas del medicamento en sí, ya sea solo o en combinación con otros medicamentos. El reconocimiento y la prevención de todo tipo de eventos adversos por medicamentos es por tanto una prioridad.

La incapacidad para reconocer una reacción adversa a un fármaco puede comprometer la salud del paciente, particularmente cuando la reacción es confundida con un síntoma de un nuevo problema de salud. Si esto se tra- 
ta posteriormente con otro fármaco, el resultado será la prescripción en cascada. Esto puede hacer que la reacción adversa inicial sea más difícil de reconocer y expone al paciente a la aparición de nuevas reacciones adversas.

\section{¿Qué es una cascada de prescripción?}

La cascada de prescripción se produce cuando se prescribe un nuevo medicamento para "tratar" una reacción adversa asociada con otro medicamento, basada esta prescripción en la creencia errónea de que una nueva condición médica que requiere tratamiento se ha instaurado (figura 1$)^{2}$. La prescripción en cascada también puede aparecer cuando nos anticipamos a la aparición de una reacción adversa. Un ejemplo es la prescripción de un inhibidor de la bomba de protones para evitar los efectos adversos gastrointestinales asociados al uso de antiinflamatorios no esteroideos (AINEs). Las cascadas de prescripción resultantes tanto de reacciones adversas conocidas como desconocidas, ponen al paciente en una situación de sufrir un daño mayor.

\section{Principales medicamentos implicados en "Cas- cada de Prescripción"}

Dentro de los fármacos ampliamente prescritos desde las consultas médicas, muchos de ellos se encuentran relacionados con la prescripción en cascada. Entre ellos se incluyen medicamentos para la demencia, ansiolíticos, antidepresivos, antiepilépticos, opioides, AINEs, antibióticos y antieméticos. En la tabla 1 se muestra algunos ejemplos de cascada de prescripción relacionados con estas fármacos.

\begin{tabular}{|c|c|c|}
\hline Fármaco & Reacción adversa & $\begin{array}{l}\text { Segundo fármaco prescrito para } \\
\text { tratar el efecto adverso del primer } \\
\text { fármaco }\end{array}$ \\
\hline Inhibidores de la colinesterasa ${ }^{15}$ & Incontinencia & Anticolinérgicos (ej.: oxibutinina) \\
\hline $\begin{array}{l}\text { Vasodilatadores, diuréticos, } \\
\text { B-bloqueantes, antagonistas del } \\
\text { calcio, IECAs, AINEs, analgésicos } \\
\text { opioides, antidepresivos, } \\
\text { ansiolíticos }^{3}\end{array}$ & Mareo & Proclorperazina \\
\hline AINEs ${ }^{2}$ & Hipertensión & Antihipertensivos \\
\hline $\begin{array}{l}\text { Diuréticos tiazídicos² } \\
\text { Metoclopramida }^{2}\end{array}$ & $\begin{array}{l}\text { Hiperuricemia, gota } \\
\text { Desórdenes del movimiento }\end{array}$ & $\begin{array}{l}\text { Alopurinol, colchicina } \\
\text { Levodopa }\end{array}$ \\
\hline IECAs 5 ,16 & Tos & Antitusígenos y/o antibióticos \\
\hline $\begin{array}{l}\text { Paroxetina, haloperidol }{ }^{17} \\
\text { Eritromicina }^{18}\end{array}$ & $\begin{array}{l}\text { Temblor } \\
\text { Arritmia }\end{array}$ & $\begin{array}{l}\text { Levodopa-carbidopa } \\
\text { Antiarrítmicos }\end{array}$ \\
\hline Antiepilépticos ${ }^{19}$ & Rash & Corticoides tópicos \\
\hline $\begin{array}{l}\text { Antiepilépticos }{ }^{19} \\
\text { Digoxina, nitratos, diuréticos del }\end{array}$ & Náuseas & Metoclopramida, domperidona \\
\hline $\begin{array}{l}\text { asa, IECAs, corticoides orales, } \\
\text { antibióticos, AINEs, analgésicos } \\
\text { opioides, metilxantinas }{ }^{20}\end{array}$ & Náuseas & Metoclopramida \\
\hline Antipsicóticos ${ }^{21}$ & Efectos extrapiramidales & Levodopa, anticolinérgicos \\
\hline
\end{tabular}

Tabla 1. Fármacos implicados en la cascada de prescripción.

Dentro de estos ejemplos de cascada de prescripción se incluye la prescripción de proclorperazina para contrarrestar el mareo inducido por medicamentos, antihipertensivos para tratar la hipertensión provocada por los AINEs y levodopa para manejar los trastornos del movimiento inducidos por la metoclopramida. Muchos otros medicamentos pueden estar involucrados en la prescripción en cascada, pero como muchas reacciones adversas son desconocidas o bien no son informadas, esta podría ser la causa de que la prescripción en cascada se encuentre infravalorada.

\section{Daño potencial}

La cascada de prescripción puede exacerbar el efecto nocivo de una reacción adversa desconocida. Un estudio llevado a cabo en Australia encontró que tras la prescripción de medicamentos que producían mareo como efecto adverso (antihipertensivos), se detectaba un aumento de la prescripción de proclorperazina ${ }^{3}$. La proclorperazina por sí misma puede inducir hipotensión postural, la cual puede exacerbar cualquier efecto hipotensivo. Esto podría explicar el aumento de la tasa de hospitalización por fractura de cadera que se observó después de que algunos pacientes comenzaron un tratamiento con proclorperazina ${ }^{3}$. Aunque el riesgo absoluto de sufrir una fractura de cadera fue pequeño, el riesgo relativo de padecer este tipo de fractura después de iniciar el tratamiento con proclorperazina se incrementó en casi un $50 \%{ }^{3}$. La fractura de cadera se asocia con elevada morbilidad y mortalidad ${ }^{4}$, destacando este hecho el potencial asociado que tiene la prescripción en cascada para provocar daños de gravedad. 
En el artículo de Liu ${ }^{5}$ se ilustra el daño potencial provocado por una cascada de prescripción en la que interviene un medicamento antihipertensivo. Una anciana desarrolla tos, que no es reconocida como causada por el IECA que está tomando. Se le prescribe un antitusígeno que contiene codeína. Cuando la tos persiste, se inicia tratamiento con antibiótico. La prescripción en cascada fue más allá, cuando el antibiótico causó diarrea asociada a Clostridium difficile. Esta cascada de prescripción culminó con la paciente hospitalizado por delirio y diarrea severa ${ }^{5}$.

\section{¿Qué factores contribuyen a la prescripción en cascada?}

Aunque los factores específicos que contribuyen a la prescripción en cascada no han sido estudiados, los factores asociados con reacciones adversas a medicamentos que pueden conducir a prescripción en cascada, sí que son bien conocidos.

Los ancianos, polimedicados y personas que utilizan medicamentos de alto riesgo, entre los que se incluyen fármacos para el sistema cardiovascular, AINEs, anticoagulantes y antibióticos, son los grupos de pacientes que presentan mayor riesgo de sufrir algún tipo de reacción adversa ${ }^{1,6,7}$.

En los ancianos, medicamentos como anticolinérgicos, antipsicóticos, benzodiacepinas, antidepresivos e hipnóticos incrementan el riesgo de aparición de reacciones adversas ${ }^{7}$. Las personas de edad avanzada pueden presentar un riesgo más elevado de sufrir prescripción en cascada que la población joven, ya que la aparición de una reacción adversa es más probable que sea confundida con la aparición de una nueva entidad clínica ${ }^{2}$. Por ejemplo, la aparición de un desorden del movimiento inducida por metoclopramida puede ser malinterpretada como enfermedad de Parkinson, pero este error en la interpretación es más difícil que se produzca en una persona joven, ya que esta enfermedad es menos prevalente durante la juventud ${ }^{2}$.
El riesgo de aparición de una reacción adversa es mayor en los primeros meses de tratamiento. Aproximadamente un $90 \%$ de los pacientes que refieren una reacción adversa, lo hacen dentro de los cuatro primeros meses de empezar el tratamiento, mientras que el $75 \%$ lo hacen dentro del primer mes $^{8}$. Muchas reacciones adversas están relacionadas con la dosis, y comenzar tratamientos con elevadas dosis en ancianos está relacionado con un incremento del riesgo de sufrir una reacción adversa ${ }^{9}$. Las reacciones adversas también pueden aparecer conforme se va incrementado las dosis.

Normalmente los pacientes no comunican ni a su médico, ni a su farmacéutico la posible aparición de una reacción adversa. Aproximadamente, un $15 \%$ de pacientes suspenden un tratamiento debido a la aparición de una reacción adversa sin comunicarlo a su médico ${ }^{10}$. Hasta un $25 \%$ de los pacientes refieren no haber recibido ningún tipo de información acerca de las posibles reacciones adversas del tratamiento que se les ha instaurado ${ }^{11}$, lo que implica que no disponen del conocimiento necesario para identificar dichas reacciones. La mala comunicación entre los profesionales de la salud y los pacientes aumenta el riesgo de aparición de reacciones adversas a medicamentos ${ }^{7}$, por lo que en la aparición de la prescripción en cascada pueden estar implicadas numerosas partes. $\mathrm{Si}$ a todo esto unimos, el aumento de la automedicación y del consumo de remedios de herboristería, tanto la comunicación efectiva entre profesionales, como la conciliación de la medicación jugarán un papel muy importante para evitar la aparición de la prescripción en cascada. La comunicación entre atención primaria y especializada será también una zona de alto riesgo, si el flujo de información entre ambos no se produce en el momento y de la manera oportuna.

\footnotetext{
Principios para la prevención de la prescripción en cascada

- Comenzar nuevos tratamientos a dosis bajas e incrementar de manera progresiva para reducir el riesgo de reacciones adversas.

- Tener en cuenta que cada nuevo síntoma pudiera ser causado por una reacción adversa, sobre todo si se ha comenzado un tratamiento nuevo o bien se ha incrementado la dosis.

- Consultar a los pacientes si han experimentado nuevos síntomas, especialmente en el caso de que se haya iniciado un tratamiento o se haya modificado una dosis.

- Proporcionar información a los pacientes sobre posibles efectos adversos de los medicamentos y qué hacer cuando aparecen dichas reacciones adversas.

- La decisión de prescribir un segundo fármaco para contrarrestar la reacción adversa provocada por un primer medicamento sólo se debe producir después de una cuidadosa valoración y cuando los beneficios de continuar la terapia con el primer fármaco superan los riesgos de las reacciones adversas adicionales causadas por el segundo fármaco.
}

Tabla 2. Principios para la prevención de la prescripción en cascada. 


\section{Prevención de la prescripción en cascada}

La prescripción adecuada consiste en la obtención del mayor beneficio terapéutico posible, con la máxima eficiencia y evitando la aparición de riesgos que el tratamiento farmacológico pueda provocar. Estos principios pueden ser aplicados para prevenir la cascada de prescripción (tabla 2).

Las reacciones adversas precipitan la cascada de prescripción, por lo que la clave para prevenir dicha cascada de prescripción es la detección temprana y evitar la aparición de reacciones adversas a medicamentos. Dado que la mayoría de las reacciones adversas a medicamentos en los ancianos están relacionadas con la dosis, comenzar el tratamiento con dosis bajas e ir valorando el efecto conforme se aumenta la dosis reducirá el riesgo de aparición de reacciones adversas.

La mayoría de reacciones adversas a medicamentos ocurren dentro de los primeros meses tras comenzar un tratamiento, por lo que los profesionales de la salud deben considerar la posibilidad de que una reacción adversa sea la causa de cualquier síntoma nuevo que aparezca, especialmente en el caso de que un medicamento nuevo se haya prescrito o bien se haya aumentado la dosis. Los profesionales de la salud deben informar a los pacientes acerca de nuevos síntomas que pudieran aparecer, ya que muchos de los pacientes no informan a su médico de la aparición de reacciones adversas.

Cuando aparecen reacciones adversas medicamentosas, utilizar estrategias no basadas en nuevos tratamientos farmacológicos pueden ser las más adecuadas como primera aproximación al manejo del paciente, en lugar de instaurar un segundo tratamiento para tratar los efectos adversos del primero. Reducir la dosis del fármaco que causa la reacción adversa es apropiado cuando la reacción es dosis-dependiente. Probar con un fármaco diferente que presente un efecto similar, pero con menos riesgo de provocar algún tipo de reacción adversa es una buena vía para evitar la prescripción en cascada. Por ejemplo, en un paciente que usa metoclopramida para el alivio de las náuseas y los vómitos y desarrolla efectos adversos extrapiramidales, lo correcto es cambiar a domperidona, un medicamento con menor riesgo de desarrollar algún tipo de desorden del movimiento.

Reconsiderar la necesidad del medicamento causante de la reacción adversa es también una estrategia adecuada. Si los riesgos asociados a continuar el tratamiento superan a los beneficios, suspender el tratamiento parece una decisión muy apropiada. La decisión de prescribir un segundo medicamento para tratar una reacción adversa provocada por el tratamiento inicial sólo se debe realizar después de un cuidadoso examen de la situación. Los beneficios de la terapia con el primer medicamento deben superan los riesgos adicionales de la reacción adversa del segundo fármaco.

A los pacientes se les debe proporcionar conocimientos e información suficientes para ayudarles a identificar reacciones adversas a medicamentos, y de este modo evitar cascadas de prescripción. Se debe incluir información que describa los posibles efectos adversos a los medicamentos y qué hacer cuando aparecen estos efectos adversos. Se hará especial hincapié en el momento en que se instaure un nuevo tratamiento, o bien cuando se aumente la dosis de fármaco. Se debe considerar la revisión de los botiquines domiciliarios, especialmente en aquellos pacientes que presentan un elevado riesgo de sufrir una reacción adversa, y por consiguiente de provocar una prescripción en cascada. Estas revisiones mejoran la identificación y resolución de problemas relacionados con los medicamentos $^{12}$, y reducen las hospitalizaciones causadas por la medicación $n^{13,14}$.

\section{DESPRESCRIPCIÓN}

Existen muchas guías basadas en la evidencia que ayudan a los médicos a comenzar un tratamiento, pero existe mucha menos información acerca de cómo retirar un fármaco o suspender un tratamiento. Varios términos se han utilizado para retirar un medicamento, entre los que se incluyen desprescripción, retirada, suspensión, fármacolisis y poda de la prescripción. Aquí desprescripción se utiliza para definir la interrupción de una terapia a largo plazo, supervisada por un médico.

\section{¿Cuándo debe ser considerada la desprescrip- ción?}

La buena práctica nos dice que debemos revisar la medicación de un paciente periódicamente. Esta revisión puede ser un buen momento para plantearnos la desprescripción.

\section{Polifarmacia}

El término polifarmacia abarca varias definiciones: a) el uso de más medicación de la clínicamente indicada; b) la utilización de 5 medicamentos o más, y c) el uso de muchos medicamentos al mismo tiempo. En las personas mayores está asociada con un mayor riesgo de deterioro de la función física y cognitiva, institucionalización, hospitalización y muerte. Estas asociaciones parecen ser independientes de las enfermedades subyacentes ${ }^{22}$. Además, varios estudios demuestran que la reducción del número de fármacos tiene efectos positivos sobre las personas mayores. Un estudio sobre viabilidad para reducir la polifarmacia en personas mayores de 70 años sugiere que casi la mitad de los medicamentos que tienen prescritos podrían ser retirados. Sólo el $2 \%$ de los fármacos retira- 
dos tuvieron que volver a prescribirse debido a que reapareció la condición original. En general, hubo una mejoría en la capacidad cognitiva y en la salud global del paciente ${ }^{23}$. Una revisión de estudios de retirada de medicación en personas de edad avanzada encontró que la retirada de dicha medicación raramente se asocia con la aparición de efectos adversos ${ }^{24}$. Después de la retirada de la terapia antihipertensiva muchos pacientes (20-85\%) permanecieron normotensos y la retirada de fármacos psicotrópicos se asoció con una disminución de caídas y una mejora de la capacidad cognitiva ${ }^{24}$. Estos datos además, son apoyados por un reciente estudio piloto que confirma la viabilidad de la desprescripción en polifarmacia ${ }^{25}$.

\section{Reacciones adversas a medicamentos}

Es de sentido común retirar un tratamiento o reconsiderar su relación beneficio/riesgo, cuando éste provoca una reacción adversa. Por ejemplo, en los ensayos clínicos en prevención secundaria del ictus con fármacos antiagregantes, más del $20 \%$ de los pacientes tienen que suspender el tratamiento debido a la aparición de efectos adversos. Dado que los participantes en dichos ensayos no reflejan a los pacientes reales, es probable que en la práctica cotidiana un mayor número de pacientes sea incapaz de tolerar estos medicamentos. Los profesionales de la salud a menudo tienen dificultad para reconocer las reacciones adversas en parte porque las desconocen $\mathrm{y}$, además, porque estas reacciones adversas pueden ser confundidas con los síntomas de la enfermedad. Las caídas y el deterioro cognitivo son frecuentemente reacciones adversas a medicamentos en las personas mayores, pero a menudo son ignoradas, siendo consideradas como parte del proceso normal de envejecimiento ${ }^{26}$.

\section{Falta de eficacia}

Para muchos fármacos no es posible evaluar su eficacia de manera rápida y directa sobre el enfermo. Esto es debido a que muchos fármacos son utilizados para prevenir enfermedades, o bien porque el número de pacientes que deben ser tratados para que el beneficio sea perceptible es demasiado grande ${ }^{27}$. De todos modos, si un fármaco no tiene efecto en una variable subrogada (p.ej.: presión arterial o colesterol total) o síntoma, no tiene sentido continuar la terapia porque estamos aumentando los costes y el riesgo de que aparezca algún daño sin obtención de beneficio alguno.

\section{Caídas}

La polifarmacia y los fármacos que actúan a nivel del sistema nervioso central son un importante factor de riesgo para las caídas, incrementando el mismo alrededor de un $50 \%{ }^{28}$. Un ensayo clínico controlado con placebo demostró que tras la re- tirada de fármacos psicotrópicos las caídas se redujeron en un $66 \%{ }^{29}$. El número de fracturas de cadera se puede reducir en un $10 \%$ simplemente evitando o disminuyendo el uso de benzodiacepinas en personas de edad avanzada ${ }^{30}$. La relación entre antihipertensivos y caídas no está establecida tan claramente como en el caso anterior. En personas mayores los fármacos antihipertensivos son retirados a menudo en el caso de aparición de hipotensión ortostática o de caídas recurrentes.

\section{Enfermedad terminal, demencia o fragilidad}

Es importante reevaluar el papel de ciertos medicamentos cuando el paciente entra en las fases terminales de la enfermedad, empieza a desarrollar signos de fragilidad o bien desarrolla una demencia incapacitante, ya que los objetivos del tratamiento no deberían ser los mismos que al comienzo del mismo. La mayoría de las terapias preventivas, como medicamentos para tratar la hipertensión, hiperlipidemias y osteoporosis necesitan muchos meses e incluso años para establecer su beneficio. Estos medicamentos tienen un valor reducido en pacientes con una expectativa de vida reducida. Por otra parte, hay que tener en cuenta que las propiedades farmacodinámicas y farmacocinéticas de muchos fármacos se ven alteradas en pacientes con enfermedades terminales, pacientes con elevado grado de fragilidad y esto influye directamente en la relación beneficio/riesgo del fármaco. La desprescripción reducirá la carga de medicación del paciente, disminuirá la aparición de efectos adversos y desplazará el enfoque terapéutico del paciente en fase terminal hacia aspectos que son más importantes para el paciente ${ }^{31}$.

\section{¿Cuáles son las consecuencias de la despres- cripción?}

Como todas las intervenciones médicas, incluyendo el inicio de un tratamiento, existen beneficios y riesgos potenciales en la desprescripción. Por lo tanto, son varios los posibles resultados que podemos obtener tras retirar algún medicamento a un paciente.

\section{Ningún cambio evidente en el estado clínico}

En muchos casos puede que no haya ningún cambio clínico evidente en el estado del paciente después de retirar un medicamento. Sin embargo, la satisfacción del paciente puede mejorar, el costo económico para el paciente y la comunidad se reduce y el riesgo de interacciones y reacciones adversas se elimina.

\section{Resolución de determinadas reacciones adver-} sas

Las reacciones adversas dosis dependientes suelen resolverse coincidiendo con la eliminación del fármaco del torrente sanguíneo (3-5 vidas medias). 
Sin embargo, en algunas situaciones, tales como el delirio, la resolución puede llevar mucho más lo esperado por cuestiones farmacocinéticas.

\section{Mejora de la función y la calidad de vida}

En un ensayo clínico destinado a reducir la polifarmacia en pacientes de edad avanzada, la escala de valoración global de los pacientes mejoró en un $88 \%$ de los mismos, y en la mayoría de ellos se recuperó la función cognitiva ${ }^{23}$. Una revisión sistemática concluyó que la retirada de fármacos puede estar asociada con una mejoría en la capacidad cognitiva y en el comportamiento en pacientes con demencia, y además disminuyó el número de caídas ${ }^{24}$. La simplificación del régimen farmacológico también mejoró la adherencia y los errores de medicación.

\section{Síndromes de retirada}

Cuando la desprescripción se lleva a cabo lentamente y bajo supervisión médica, es muy rara la aparición de reacciones adversas clínicamente significativas relacionadas con la retirada del medicamento $^{23,24}$. Aún así, los médicos deberían ser conscientes de la posible aparición de problemas relacionados con la retirada del fármaco.

\section{Síndromes de retirada y discontinuación}

La causa más común de aparición de síndromes de discontinuación es la retirada de fármacos que actúan sobre el sistema nervioso central. La mayoría de los profesionales de la salud deberían estar familiarizados con el síndrome de discontinuación que aparece tras la retirada de los inhibidores selectivos de la recaptación de serotonina. Los síntomas suelen aparecer dentro de la primera semana tras retirar el fármaco. Suelen ser leves, y se resuelven

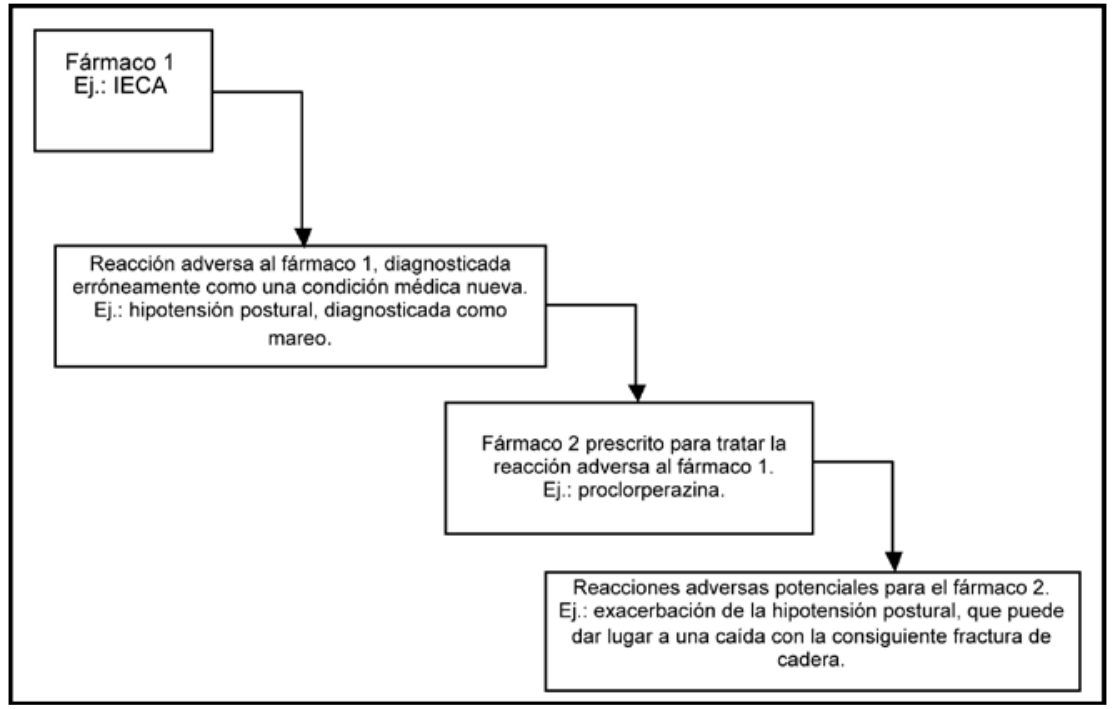

Figura 1. La cascada de prescripción (adaptado de Rochon²).

en un plazo de alrededor de diez días. La retirada rápida y no escalonada de las benzodiacepinas se asocia con un síndrome de retirada mucho más serio, incluyendo confusión, alucinaciones y convulsiones. Cuando la levodopa es retirada de manera abrupta, aparece un síndrome de abstinencia grave con características de síndrome neuroléptico maligno, incluyendo rigidez muscular severa, inestabilidad autonómica y alteración de la consciencia. En pacientes que han estado tomando corticoides sistémicos durante varias semanas, la retirada repentina del fármaco puede conducir a veces a la aparición de una crisis Addisoniana secundaria a la supresión del eje hipotalámico-hipofisario-adrenal $^{32,33}$.

\section{Síndromes de rebote}

La retirada brusca de los B-bloqueantes se asocia con taquicardia e hipertensión de rebote que puede agravar la insuficiencia cardiaca y la cardio- patía isquémica.

La retirada de los inhibidores de la bomba de protones tras un largo periodo de tratamiento se asocia con hipersecreción ácida de rebote y agravamiento de los síntomas gastrointestinales ${ }^{33}$. Las gotas nasales y los analgésicos simples pueden obtenerse sin receta fácilmente, pero la cesación puede asociarse respectivamente con rinorrea y dolor de cabeza $^{34}$. La aparición de insomnio de rebote es común después de la suspensión del tratamiento con fármacos hipnóticos.

\section{Reaparición de los síntomas de la enfermedad original o factor de riesgo}

Es importante no malinterpretar un síndrome de rebote o retirada como un resurgimiento de los síntomas de la original enfermedad. Sorprendentemente, los estudios sobre retirada de fármacos no muestran una alta incidencia de síntomas de la 


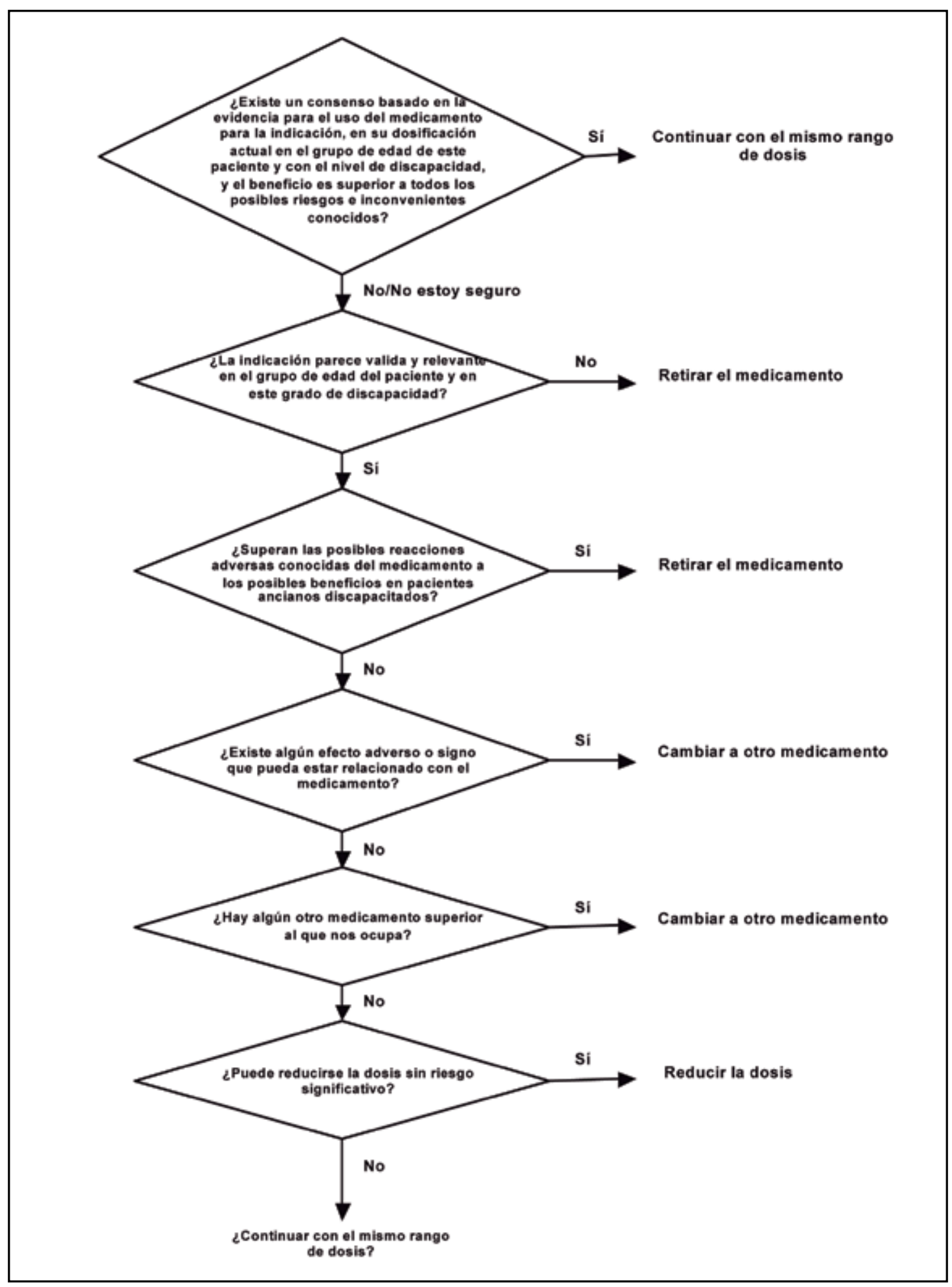

Figura 2. Algoritmo de desprescripción (adaptado de Villafaina ${ }^{35}$ ).

enfermedad original o factores de riesgo que reaparecen tras la detención del tratamiento ${ }^{24}$. Sin embargo, si algunos fármacos, por ejemplo inmunosupresores, se detienen abruptamente la condición subyacente puede empeorar.

¿Cómo debemos enfocar la desprescripción?

Existe evidencia clínica limitada para guiarnos en 
el proceso de la desprescripción, pero se pueden aplicar algunos principios generales (figura 2) ${ }^{35}$.

\section{Preparar al paciente para la desprescripción}

Cuando se inicia un tratamiento, se debe explicar al paciente que el resultado del mismo será supervisado, y que el fármaco podría ser retirado si no se obtiene un efecto beneficioso o bien aparece un efecto adverso clínicamente importante. Las expectativas del paciente deben ser discutidas y consensuadas en ese momento. Esto es particularmente importante para medicamentos como los inhibidores de la colinesterasa para la enfermedad de Alzheimer, en los que los beneficios o mejoría clínica se deben confirmar por un especialista, ya que en determinados países de este informe dependerá que se continúe con la financiación de estos medicamentos.

\section{Reconocer la necesidad de desprescribir}

Las principales situaciones en las que la desprescripción puede ser útil son la polifarmacia, las reacciones adversas a medicamentos (p.ej.: caídas en personas ancianas), la pérdida de eficacia del tratamiento, los cambios en los objetivos del tratamiento que pudieran estar asociados a la aparición de enfermedad terminal, demencia o fragilidad.

\section{Priorizar fármacos a retirar o dosis a reducir}

Un enfoque cauteloso es retirar o reducir la dosis de un sólo medicamento en cada momento. Esto ayuda a identificar qué medicamento puede ser el responsable del daño, o si aparece síndrome de retirada conocer qué medicamento exactamente debe ser readministrado. Si lo que sospechamos es una reacción adversa a un medicamento, obviamente dicho fármaco es el primero que debería ser retirado.

Si el paciente es anciano y toma múltiples medicamentos, entonces existen varios enfoques posibles para identificar los fármacos que son adecuados para desprescribir, centrándose en particular en los medicamentos anticolinérgicos y en los que presentan algún tipo de efecto sedante ${ }^{23,36,37}$. Reducir la dosis de un medicamento o cambiar el patrón de fármaco de consumo pautado a fármaco de consumo a demanda también podría ser una estrategia adecuada.

\section{Disminución gradual de la dosis}

En muchos casos, los medicamentos pueden ser retirados de manera brusca sin someter al paciente a un riesgo excesivo, sin embargo existen otros grupos de medicamentos (B-bloqueantes, corticosteroides, benzodiacepinas, opioides, levodopa) en los que la retirada repentina de la medicación puede generar graves síndromes de retirada/absti- nencia o de rebote. La duración de la disminución gradual de la dosis puede variar de días a meses, y está influenciada por diferentes factores como la vida media del fármaco, disponibilidad de diferentes dosificaciones del medicamento, así como la respuesta fisiológica y psicológica del paciente. Para fármacos psicotrópicos, el objetivo general podría ser reducir la dosis un $25 \%$ cada mes, ajustando ésta a la respuesta del paciente ${ }^{23,38,39}$. Se trata de un régimen razonablemente conservador que sería adecuado para muchos otros medicamentos, en los que sería preocupante la aparición de un síndrome de retirada o rebote.

En pacientes que han estado tomando benzodiacepinas durante un largo periodo, podría ser beneficioso el intercambio de la dosis de la citada benzodiacepina en la dosis equivalente de diazepam, debido a su larga vida media, comenzando después la retirada. Sin embargo, en los pacientes que han tenido una reacción adversa grave, lo normal sería retirar el fármaco inmediatamente.

\section{Monitorización de los resultados}

Si se produce un síndrome de retirada o rebote de importancia, se debe reanudar la administración del medicamento. La retirada se debería intentar más tarde a un ritmo más lento.

Es necesario evaluar a los pacientes para verificar los resultados positivos de la desprescripción, tales como disminución de los efectos adversos y mejora de la función global del paciente. Estos beneficios son importantes a la hora de continuar con el cumplimiento o no de la retirada.

\section{CONCLUSIÓN}

La cascada de prescripción tiene la capacidad de ocasionar daños graves a los pacientes. Los prescriptores deben ser conscientes del potencial de los fármacos para causar efectos adversos, particularmente en los ancianos y en pacientes que utilizan medicamentos comúnmente asociados con la aparición de reacciones adversas. Es necesaria una mejora en la capacidad de reconocimiento de que ciertas reacciones adversas pueden conducir a la prescripción en cascada.

Reconocer los resultados adversos o la falta de eficacia requiere habilidad y diligencia, especialmente en personas mayores polimedicadas. Sin embargo, el buen cuidado del paciente depende de la capacidad del médico prescriptor para evaluar la necesidad clínica de desprescribir un fármaco y luego llevar a cabo la retirada supervisada de medicamentos cuando sea necesario.

\section{BIBLIOGRAFÍA}


1. Miller GC, Britth HC, Valenti L. Adverse drug events in general practice patients in Australia. Med $\mathrm{J}$ Aust. 2006;184:321-4.

2. Rochon PA, Gurwitz JH. Optimising drug treatment for elderly people: the prescribing cascade. BMJ. 1997; 315:1096-9.

3. Caughey GE, Roughead EE, Pratt N, Shakib S, Vitry Al, Gilbert AL. Increased risk of hip fracture in the elderly associated with prochlorperazine: is a prescribing cascade contributing? Pharmacoepidemiol Drug Saf. 2010; 19:977-82.

4. Bentler SE, Liu L, Obrizan M, Cook EA, Wright KB, Geweke $\mathrm{JF}$, et al. The aftermath of hip fracture: discharge placement, functional status change, and mortality. Am J Epidemiol. 2009; 170:1290-9.

5. Liu PT, Argento VS, Skudlarska BA. Prescribing cascade in an 80-year-old Japanese immigrant. Geriatr Gerontol Int. 2009; 9:402-4.

6. Burgess CL, Holman CD, Satti AG. Adverse drug reactions in older Australians, 1981-2002. Med J Aust. 2005; 182:267-70.

7. Easton $\mathrm{K}$, Morgan T, Williamson M. Medication safety in the community: a review of the literature. Sydney: National Prescribing Service; 2009. www.nps.org.au/research_and_ evaluation/current_research/medication_safety_community [cited 2011 Nov 7]

8. Colebatch KA, Marley J, Doecke C, Miles H, Gilbert A. Evaluation of a patient event report monitoring system.Pharmacoepidemiol Drug Saf. 2000; 9:491-9.

9. Routledge PA, O'Mahony MS, Woodhouse KW. Adverse drug reactions in elderly patients. $\mathrm{Br} \mathrm{J}$ Clin Pharmacol. 2004; 57:121-6.

10. Blendon RJ, Schoen C, DesRoches C, Osborn R, Zapert K. Common concerns amid diverse systems: Health care experiences in five countries. Health Aff (Millwood). 2003; 22:106-21.

11. Schoen C, Osborn R, Huynh PT, Doty M, Davis K, Zapert K, et al. Primary care and health system performance: Adults' experiences in five countries. Health Aff. 2004;Suppl web exclusives:W4-487-503.

12. Gilbert AL, Roughead EE, Beilby J, Mott K, Barratt JD. Collaborative medication management services: improving patient care. Med J Aust. 2002; 177:189-92.

13. Roughead EE, Barratt JD, Ramsay E, Pratt N, Ryan P, Peck $\mathrm{R}$, et al. The effectiveness of collaborative medicine reviews in delaying time to next hospitalisation for patients with heart failure in the practice setting: results of a cohort study. Circ Heart Fail. 2009; 2:424-8.

14. Roughead EE, Barratt JD, Ramsay E, Pratt N, Ryan P, Peck $\mathrm{R}$, et al. Collaborative home medicines review delays time to next hospitalization for warfarin associated bleeding in Australian war veterans. J Clin Pharm Ther. 2011; 36:27-32.

15. Gill SS, Mamdani M, Naglie G, Streiner DL, Bronskill SE, Kopp A, et al. A prescribing cascade involving cholinesterase inhibitors and anticholinergic drugs. Arch Intern Med. 2005; 165:808-13.

16. Vegter S, de Jong-van den Berg LT. Misdiagnosis and mistreatment of a common side-effect-angiotensin-converting enzyme inhibitor-induced cough. $\mathrm{Br} \mathrm{J}$ Clin Pharmacol. 2010; 69:200-3

17. Mallet L, Spinewine A, Huang A. The challenge of managing drug interactions in elderly people. Lancet. 2007;370:18591.

18. Corrao G, Botteri E, Bagnardi V, Zambon A, Carobbio A, Falcone $\mathrm{C}$, et al. Generating signals of drug-adverse effects from prescription databases and application to the risk of arrhythmia associated with antibacterials. Pharmacoepidemiol Drug Saf. 2005; 14:31-40.
19. Tsiropoulos I, Andersen M, Hallas J. Adverse events with use of antiepileptic drugs: a prescription and event symmetry analysis. Pharmacoepidemiol Drug Saf. 2009; 18:48391.

20. Bytzer P, Hallas J. Drug-induced symptoms of functional dyspepsia and nausea. A symmetry analysis of one million prescriptions. Aliment Pharmacol Ther. 2000; 14:1479-84.

21. Rochon PA, Gurwitz JH. Drug therapy. Lancet. 1995; 346:32-6.

22. Hilmer SN, Gnjidic D. The effects of polypharmacy in older adults. Clin Pharmacol Ther. 2009; 85:86-8.

23. Garfinkel D, Mangin D. Feasibility study of a systematic approach for discontinuation of multiple medications in older adults: addressing polypharmacy. Arch Intern Med. 2010; 170:1648-54.

24. Iyer S, Naganathan V, McLachlan AJ, Le Couteur DG. Medication withdrawal trials in people aged 65 years and older: a systematic review. Drugs Aging. 2008; 25:1021-31.

25. Beer C, Loh PK, Peng YG, Potter K, Millar A. A pilot randomized controlled trial of deprescribing. Ther Adv Drug Saf. $2011 ; 2: 37-43$.

26. Hilmer SN, McLachlan AJ, Le Couteur DG. Clinical pharmacology in the geriatric patient. Fundam Clin Pharmacol. 2007; 21:217-30.

27. Le Couteur DG, Kendig H. Pharmaco-epistemology for the prescribing geriatrician. Aust J Ageing. 2008; 27:3-7.

28. Boyle N, Naganathan V, Cumming RG. Medication and falls: risk and optimization. Clin Geriatr Med. 2010; 26:583-605.

29. Campbell AJ, Robertson MC, Gardner MM, Norton RN, Buchner DM. Psychotropic medication withdrawal and a home-based exercise program to prevent falls: a randomized, controlled trial. J Am Geriatr Soc. 1999; 47:850-3.

30. Cumming RG, Le Couteur DG. Benzodiazepines and risk of hip fractures in older people - a review of the evidence. CNS Drugs. 2003; 17:825-37.

31. Holmes HM. Rational prescribing for patients with a reduced life expectancy. Clin Pharmacol Ther. 2009; 85:1037.

32. Woodward MC. Deprescribing: achieving better health outcomes for older people through reducing medications. $J$ Pharm Pract Res. 2003; 33:323-8.

33. Proton-Pump Inhibitor Therapy Induces Acid-Related Symptoms in Healthy Volunteers After Withdrawal of Therapy. Gastroenterology. 2009; 137:80-7.

34. A practical guide to stopping medicines in older people. Best Pract J. 2010; 27:10-23. www.bpac.org.nz/magazine/2010/april/stopGuide.asp [cited 2011 Nov 8].

35. Villafaina A, Gavilán E. Pacientes polimedicados frágiles, un reto para el sistema sanitario. Información Terapéutica del Sistema Nacional de Salud. 2011; 35(4);114-23.

36. Hilmer SN, Mager DE, Simonsick EM, Cao Y, Ling SM, Windham BG, et al. A drug burden index to define the functional burden of medications in older people. Arch Intern Med. 2007; 167:781-7.

37. Fick DM, Cooper JW, Wade WE, Waller JL, Maclean JR, Beers $\mathrm{MH}$. Updating the Beers criteria for potentially inappropriate medication use in older adults: results of a US consensus panel of experts. Arch Intern Med. 2003; 163:2716-24.

38. Parr JM, Kavanagh DJ, Cahill L, Mitchell G, McD Young R. Effectiveness of current treatment approaches for benzodiazepine discontinuation: a meta-analysis. Addiction. 2009; 104:13-24.

39. Lader M. Pharmacotherapy of mood disorders and treatment discontinuation. Drugs. 2007; 67:1657-63. 